CASE REPORT

\title{
Combination of Encephalo-Myo-Pial-Synangiosis and Encephalo- Arterio-Pial-Synangiosis Procedure in Pediatric Moya-Moya Disease
}

\author{
Prastiya Gunawan $^{1 *}$, Wihasto Suryaningtyas ${ }^{2}$, Darto Saharso ${ }^{1}$, Risky Prasetyo ${ }^{1}$
}

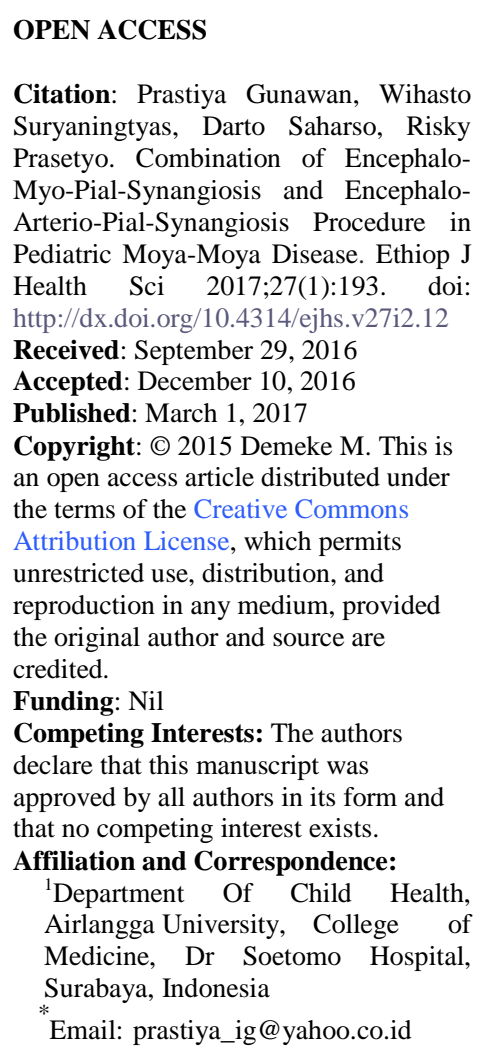

\section{OPEN ACCESS}

Citation: Prastiya Gunawan, Wihasto Prasetyo Combination of EncephaloHealth Sci http://dx.doi.org/10.4314/ejhs.v27i2.12

Received: September 29, 2016

Published: March 1, 2017

Copyright: () 2015 Demeke M. This is an open access article distributed under Attribution License, which permits unrestricted use, distribution, and theduction in any medium, provided credited. approved by all authors in its form an that no competing interest exists.

${ }^{1}$ Department Of Child Health,

Medicine Dr Soetomo Hospital,

Surabaya, Indonesia

Email: prastiya_ig@yahoo.co.id

\begin{abstract}
BACKGROUND: Moya-moya disease in children is a cerebrovascular disorder that may cause cerebral ischemic or hemorrhage.

CASE DETAILS: We report an 8-year-old boy that was admitted with the chief complaint of repeated sudden half left body paralyze. MRA showed acute thrombotic infarction in the right hemisphere and internal carotid artery stenosis in the form of puffs of smokes. Indirect revascularization surgical procedure with combination of Encephalo-myo-pial-synangiosis (EMS) and Encephalo-arterio-pial-synangiosis (EAS) was performed. It resulted in a good response.

CONCLUSION: Moya-moya disease can lead to permanent neurological disability if untreated. Satisfactory outcome was noted following combination surgery management with EMS and EAS.

KEYWORDS: Moya-moya disease, Encephalo-myo-pialsynangiosis, Encephalo-arterio-pial-synangiosis, children

\section{INTRODUCTION}

Moyamoya disease (MMD) is a rare unremitting cerebrovascular occlusive disorder (1). The etiology and pathogenesis remain unknown (2). The presentations generally are ischemic warning sign in children and hemorrhage symptoms in adults (3). It can lead to a devastating, permanent neurological disability if untreated (2).

Moyamoya disease may cause cerebral ischemia (transient ischemic attacks or infarction) and sometimes haemorrhage (intracerebral,intraventricular andsubarachnoid). Headache, involuntary movement and convulsive seizures may also occur (1).

The purpose of this paper is to report a case of Moyamoya disease in a child, focusing on diagnosis and management. 


\section{CASE REPORT}

An 8-year-old boy was admitted to Dr Soetomo Hospital in the year 2015 with the chief complaint of sudden left half body paralyze after awakening. There was mild impairment of alertness following the weakness, and it repeated in a period of 15 to 30 days since the boy was 3 years old. Each episode lasted for five minutes and was preceded by headaches.

He was presented with aphasia, facial asymmetries and left ptosis. Physiologic reflexes were increased, and pathological reflexes were positive. Head Magnetic Resonance Imaging (MRI) showed infarction with encephalomalacia cyst in right temporal region (Figure 1). Head Magnetic Resonance Angiography (MRA) revealed : (1) Acute thrombotic infarction at the subcortical multiple frontoparietal right hemisphere, the right nucleus caudate, the right nucleus lentiform, and the right corona radiate; (2) Internal Carotid Artery (ICA) stenosis along the left and the right branches until the whole right and left middle cerebral artery (MCA) and the anterior cerebral artery (ACA) (with small collateral arteries) in the lenticularstriatal area (basal ganglia) in the form of puffs of smokes; (3) Right and left Posterior Cerebral Artery (PCA) stenosis accompanied by small collateral arteries. The MRA supported Moyamoya disease (Figure 2).

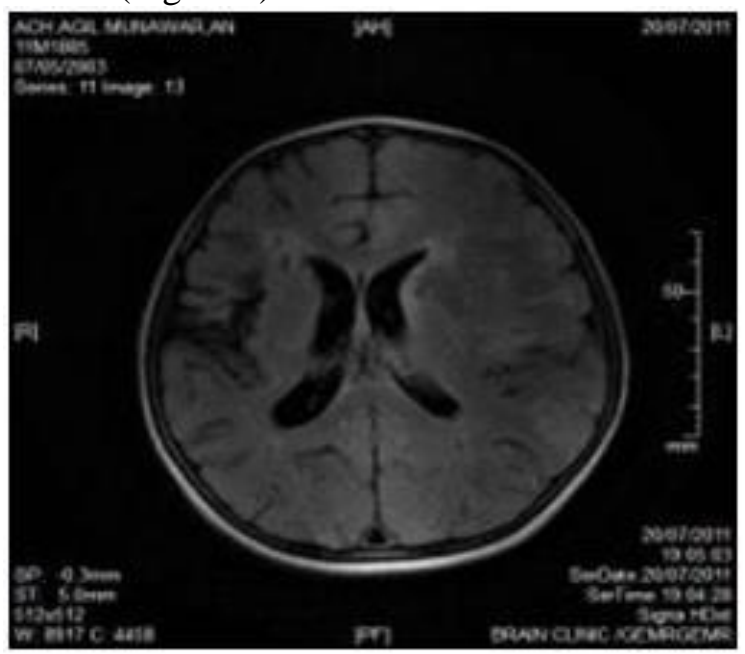

Figure 1: Head MRI showing infarction in right temporal region and encephalomalacia cyst in right temporal region

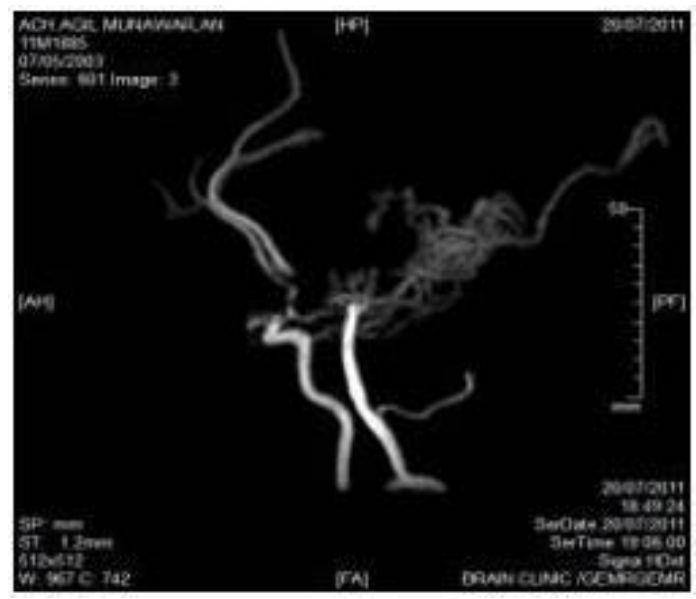

Figure 2: Head MRA showing puff of smokes

Indirect revascularization surgical procedure was performed. Combination of Encephalomyo-pial-synangiosis (EMS) and Encephaloarterio-pial-synangiosis (EAS) was chosen over other techniques due to its relatively easy-to-do technique while it still gives and satisfying result. After physical rehabilitation in several weeks, the patient was discharged with a relatively good condition leaving neurological sequalae.

\section{DISCUSSION}

Moyamoya disease should be considered among Asian children presenting with stroke (4). Commonly, MMD presents with acute neurologic deficits or unexplained symptoms such as cerebral ischemia, hemiparesis, aphasia, dysarthria and cognitivedisturbance (2). Initially, most children with MMD have extremity weakness or paralysis with or without alternating hemiplegia (1). In our case, the boy suffered from sudden left side paralysis, impairment of consciousness, aphasia, involuntary movement and convulsive seizures leading to stroke-like episodes.

Neurologic deficits are extensive and diverse in MMD. The attacks are likely to occur after crying, coughing, or blowing on hot noodles, all of which may cause hyperventilation developing in constriction of cerebral arteries (5).

In this case, head MRA supported the MMD. The diagnosis of MMD is created based

DOI: http://dx.doi.org/10.4314/ejhs.v27i2.12 
on radiographic findings including restricting of the terminal segments of the ICA, frequently with the associated development of collateral vessels (6). Cerebral angiography remained the gold standard. MRA has been recommended as a definitive noninvasive diagnostic procedure for children (7).

Moyamoya Disease often presents with quick onset and offset of hemiparesis. Recurrent episode of hemiplegia typically involves one side of the body. Persistent unilateral slowing on the EEG following hyperventilation suggests the disorder $(2,7)$.

This condition warrants a surgical procedure to revascularize the ischemic brain. Medical treatment does not appear to be beneficial. Revascularization should be considered in nonemergent situations (8). Advanced neurologic deficits such as cognitive decline or progressive seizures are indications for the bypass. As the bypass surgery, direct revascularization may not always give the best results; there are other indirect revascularization techniques which could be achieved with higher success degree and reduced complications (1-3).

The recent combination of indirect revascularization technique gives a better result than one technique alone. Surgical EMS and EAS procedure were performed in this patient. The superficial temporal artery (STA) is disjoined at its proximal section and separated from the inner surface of the skin. The galea is incised aligned to the STA to offer a cuff of tissue above the exposed length of the vessel. Then, it is dissected thoroughly away and separated from the fascia below, along with the point at which the artery crosses the incised skin. The temporalis muscle is isolated from the bone with a periosteal elevator and retracted posteriorly with the STA. Craniotomy procedure was introduced by linking two burrholes, inferior temporal burrhole-beneath the proximal part of the STA further, with the other one, superior temporal-below distal part of the STA along with the concern not to injure the dural vasculature. A cross durotomy was made along the course of the STA. Arachnoid membrane covering the brain was unclosed along the sulci as much as possible, and the STA were anchored to the pia with several stitches of
7-0 prolene. The dural opening was then sealed with a single flap of the superficial temporal muscle. Through this procedure, combine EAS and EMS have been done (9-11). Angiographic modality studies for the evaluation should be performed ranged from 9 days to 6 months after the surgery; collateral circulation was mainly provided through the direct bypass procedure (911).

The prognosis of MMD was difficult to predict. The progression of disease can be restricted with rare intermittent events, or can be uncontrollable with rapid neurologic decline (1). However, the prognosis of this case is good.Yet, the risk of stroke can occur within the first 30 days after surgery (approximately $4 \%$ per hemisphere) (1). Blood pressure should be monitored for the first 24 hours due to the risk of bleeding, thrombosis and occlusion. Any deviations in neurologic examination should be evaluated with emergency CT.

In conclusion, the diagnosis of moya-moya was established on clinical evidence supported with the neuroimaging that showed a puff of smokes. A combination of encephalo-myosinangiois and encephalo-artery-sinangiois surgery and physical therapy resulted in a good response.

\section{ACKNOWLEDGEMENT}

The author would thank to Mrs Nur Fitriyah for English editing.

\section{REFERENCES}

1. Smith JL. Understanding and treating moyamoya disease in children. Neurosurg focus 2009;26:1-11.

2. Scott RM, Smith ER. Moyamoya disease and moyamoya syndrome. NEJM 2009;360:1226-37.

3. Baaj AA, Agazzi S, Sayed ZA, Toledo M, Spetzler RF, van Loveren H. Surgical management of moyamoya disease: a review. Neurosurg focus 2009;26:1-7.

4. Achrol AS, Guzman R, Lee M, Steinberg GK. Pathophysiology and genetic factors in moyamoya disease. Neurosurg focus 2009;26:1-6. 
5. Janda PH, Bellew JG, Veerappan V. Moyamoya disease: case report and literature review. JAOA 2009;109:547-53.

6. Demartini Jr Z, Martins RT, Rocha CE, Matos LA, Spotti AR, Tognola WA, et al. Surgical treatment of moyamoya disease in children. Arq Neuropsiquiatr 2008;66:276.

7. Phi JH, Wang KC, Lee JY, Kim SK. Moyamoya Syndrome: A Window of Moyamoya Disease. JKNS 2015;57:408-14.

8. Ishii K, Fujiki M, Kobayashi H. Invited article: surgical management of Moyamoya disease. Turk neurosurg 2008;18:107-13.

9. Thines L, Petyt G, Aguettaz P, Bodenant M, Himpens FX, Lenci $H$, et al. Surgical management of Moyamoya disease and syndrome: Current concepts and personal experience. Rev Neurol (Paris) 2015;171:31-44.

10. Patel NN, Mangano FT, Klimo P. Indirect Revascularization Techniques for Treating Moyamoya Disease. Neurosurg Clin $\mathrm{N} \mathrm{Am}$ 2010;21(3):553-63.

11. Matsushima T, Inoue TK, Suzuki SO, Inoue T, Ikezaki K, Fukui M, et al. Surgical techniques and the results of a frontotemporo-parietal combined indirect bypass procedure for children with Moyamoya disease: a comparison with the results of encephalo-duro-arterio- synangiosis alone. Clin Neurol Neurosurg 2016;12(99):S1237. 